\title{
A SURVEY OF CONTENTION BASED MEDIUM ACCESS CONTROL (MAC) PROTOCOLS IN WIRELESS LAN
}

\author{
Vigneswara Rao Gannapathy ${ }^{1}$, Lim Kim Chuan ${ }^{2}$, Siva Kumar Subramaniam², \\ Zahriladha Bin Zakaria ${ }^{4}$, Mohamad Kadim Bin Suaidi ${ }^{5}$ \\ ${ }^{1}$ Senior Lecturer, Department of Electronics and Computer Engineering, Universiti Teknikal Malaysia Melaka, \\ Melaka (UTeM), Malaysia \\ ${ }^{2}$ Senior Lecturer, Department of Electronics and Computer Engineering, Universiti Teknikal Malaysia Melaka, \\ Melaka (UTeM), Malaysia \\ ${ }^{3}$ Senior Lecturer, Department of Electronics and Computer Engineering, Universiti Teknikal Malaysia Melaka, \\ Melaka (UTeM), Malaysia \\ ${ }^{4}$ Associate Professor, Department of Electronics and Computer Engineering, Universiti Teknikal Malaysia Melaka, \\ Melaka (UTeM), Malaysia \\ ${ }^{5}$ Professor, Department of Electronics and Computer Engineering, Universiti Malaysia Sarawak (UNIMAS)
}

\begin{abstract}
In wireless network, all radio nodes are tuned to the same frequency to interconnect and establish communication between each other. All nodes in the network broadcasts their packets over a common medium and in such scenario collisions are considered as instinctive attribute. Therefore, a proper method/regulation known as Medium Access Control (MAC) protocol is required to regulate and manage an efficient access to the common channel. The protocol is designed to allow radio nodes in wireless network to broadcast their packets in an orderly and efficient manner to eliminate the collision among them. It also provides a fair bandwidth sharing to all contending nodes in the network. To date, various MAC protocols was developed to regulate the communication access among all radio nodes in wireless network. This article presents an exhaustive survey of existing contention based MAC protocols, their operations, advantages and disadvantages. Other than that, a typical MAC protocol used in IEEE 802.11 wireless networks standard, such as Carrier Sense Multiple Access (CSMA) and Carrier Sense Multiple Access with Collision Avoidance (CSMA/CA) also explained and presented in this article.
\end{abstract}

Keywords: Medium Access Control (MAC), MACA, MACAW, FAMA, Random Access Protocol, Contention Based Protocol, Binary Exponential Back-off(BEB), Virtual Carrier Sensing, Network Allocation Vector (NAV)

\section{INTRODUCTION}

The MAC protocol also enforces a fair sharing and provides equal right to all nodes in a network to access to the channel. IEEE 802.11 (IEEE. 2003) defined the standard of the MAC and physical layer protocols which are exist in the literature. To date, various MAC protocols are developed to regulate the communication in wireless network.

Contention based protocols was designed to provide efficient contention among the nodes in the network. In a contention based MAC protocol, all nodes contend for access to share the wireless channel. A packet transmission is considered successful when only one node is committing into the transmission at one time. If a simultaneous transmission is occurred on the channel (i.e. more than one node committing into transmission), then it will leads to the collision. In order to avoid such collisions, the contention resolution algorithm is invoked. The role of the contention resolution algorithms is very important in effectively utilizing the channel resources.
In order to address well known issues in wireless network (such as hidden and exposed terminal issues), the research community proposed number of reservation based collision resolution MAC protocols. This reservation based collision resolution protocols was introduced to eliminate the hidden node problem and to alleviate the exposed node problem. The protocol is required to exchange signaling packets (handshakes) before begin the DATA packet transmission. Sometimes it is referred as virtual carrier sensing where the transmitter and receiver of each node on the network will performs and exchange of control or signaling packets to reserve the channel prior to commencement of DATA packet transmission $[15,16,17,18]$.

Based on this mechanism, many well-known protocols has proposed. The Carrier Sense Multiple Access with Collision Avoidance (CSMA/CA) protocol has been selected by IEEE 802.11 standard to be used in wireless LANs [1]. CSMA/CA is random access protocol which was derived from Floor Acquisition Multiple Access (FAMA) which was presented by Fullmer [2]. This protocol is an enhanced 
version of Multiple Access with Collision Avoidance for Wireless LANs (MACAW) which was presented by [3]. MACAW protocol is based on Multiple Access with Collision Avoidance (MACA) protocol which is originally proposed by [4].

\section{CONTENTION BASED MAC PROTOCOLS}

\subsection{Multiple Access with Collision Avoidance} (MACA)

MACA protocol was proposed as an improvement of CSMA to eliminate the hidden node problem. This protocol is designed to perform handshake (i.e. exchange of control or signaling packets) between a sender node and receiver node prior initiating its DATA packet. This handshake efficiently controls the shared channel by ensuring the neighboring nodes aware on upcoming transmission and refrain from sending any packet during entire ongoing transmission.

This protocol [4] relies on control packets namely Request to Send (RTS) and Clear to Send (CTS) control packets to avoid collisions without using carrier sensing. According to this protocol the sender node initiates the transmission by transmitting a RTS control packet to inform its request to access the channel. Neighboring nodes in the vicinity of the sender node will be notified of upcoming packet transmission through this RTS control packet. Upon receiving a RTS, the receiver will respond with CTS control packet to inform its readiness to receive the DATA packet. Neighboring nodes in the range of the receiver node will be notified of upcoming packet reception through this CTS control packet.

The neighboring nodes which overhearing RTS/CTS control packets will defer themselves from sending out any packet during the ongoing transmission and wait until it's complete his transmission. This is a virtual carrier sensing and also referred as Network Allocation Vector (NAV) [4].

The MACA access method can be depicted as in Figure 1. After the exchange of RTS/CTS successfully completed, the DATA transmission from sender node to receiver node will proceeds without collision. The sequence of exchange happens between communication nodes looks like RTSCTS-DATA. If the collisions occurred among RTS control packets, then the both nodes reschedule their transmission after wait for some predefined time decided by back-off mechanism. By reducing the possibility of collision among DATA packet and eliminating the hidden node problem, MACA is proved to provide better performance compare to basic CSMA protocol.

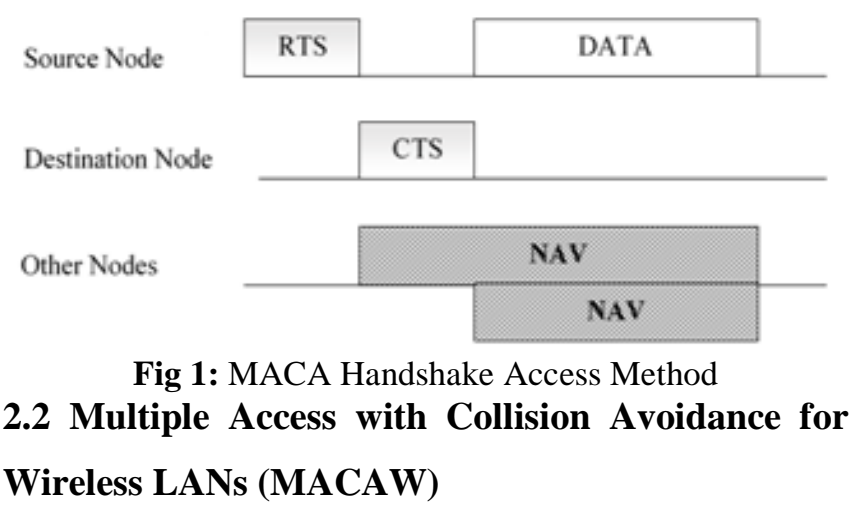

The MACA protocol was modified and a protocol called as MACAW was proposed. This protocol was introduced to solve unreliability issue which is face in MACA protocol. This was done by allowing the receiving node to acknowledge its successful DATA reception to sender node with the packet called as ACK. This is to provide reliable delivery of packet transmission that is very crucial in wireless networks. The exchange sequence of MACAW protocol is RTS-CTS-DATA-ACK [3].

This protocol was further enhance by adding Data Sensing (DS) frame in exchange sequence. The exchange sequence with the added DS frame is RTS-CTS-DS-DATA-ACK. The DS control packet is used to inform the nodes that the RTS-CTS control packets is successful exchanged. This to prevent exposed nodes to initiates their transmission. The MACAW access method can be depicted as in Figure 2.

\begin{tabular}{|c|c|c|c|c|c|}
\hline Source Node & RTS & & DS & DATA & \\
\hline Destination Node & & CTS & & & ACK \\
\hline \multirow[t]{2}{*}{ Other Nodes } & & \multicolumn{4}{|c|}{ NAV (RTS) } \\
\hline & & & \multicolumn{3}{|c|}{ NAV (CTS) } \\
\hline
\end{tabular}

Fig 2: MACAW Handshake Access Method

\subsection{Floor Acquisition Multiple Access (FAMA)}

FAMA is the enhanced version of MACA which was proposed by adding carrier sensing before sending a RTS. Every each node which is implemented with this protocol need to sense the floor ("channel") before it initiates its transmission. The protocol used both carrier sensing and RTS-CTS to sense the floor and reserve the channel for upcoming transmission respectively [2].

The node with packet will begin its transmission upon floor is sensed idle. If the busy floor is detected (i.e. fail to acquire the floor), it will defers its transmission and reschedule its bid for floor. This will be done by initializing back off mechanism or different persistence strategic.

After successfully acquired the floor, the node which is implemented with FAMA protocol, will begin its transmission by sending RTS control packet to the recipient 
node. Once the destination node successfully received the RTS control packet, then it will respond CTS control packet to the sender node. The sender node will initiate the DATA packet transmission once it received the CTS control packet. All other nodes which overhearing RTS/CTS control packets will defer from sending out any packet until the predefined transmission period which as indicated in duration field of control packets is completed. Thus, by using RTS and CTS control packets in FAMA protocol, the hidden node problem can be solved accordingly. Once the floor acquired and the channel reserved, the DATA transmission can be done successfully without any collision. The FAMA access method can be depicted as in Figure 3.

\begin{tabular}{|c|c|c|c|c|}
\hline Source Node & Acquire Channel & RTS & & DATA \\
\hline Destination Node & & & CTS & \\
\hline \multirow[t]{2}{*}{ Other Nodes } & & & \multicolumn{2}{|c|}{ NAV (RTS) } \\
\hline & & & & NAV (CTS) \\
\hline
\end{tabular}

Fig 3: FAMA Floor Acquire Access Method

\subsection{IEEE 802.11 Distributed Coordination Function (DCF) MAC Protocol}

Distributed Coordination Function (DCF) is the fundamental mechanism used to access the channel in the 802.11 MAC protocol. On the other hand, the optional Point Coordination Function (PCF) is used to support collision free and time bounded services in centralized MAC protocol. Even though these two mechanisms have differences in term of its operations, the primary concern of these two mechanisms is provides an equal right to the nodes to access the channel for successful transmission. Since PCF is not widely employed in multihop communication, the investigation in this article limited only to the DCF scheme.

Almost all IEEE 802.11 - compliant products uses DCF as MAC mechanism. At present, IEEE 802.11 DCF MAC is one of most extensively implemented MAC protocol for wireless communication. This protocol uses both collision avoidance and contention resolution schemes. It is employed with Binary Exponential Backoff (BEB) algorithm to regulate the access to the channel (contention resolution scheme). In addition, it relies on Carrier Sense Multiple Access with Collision Avoidance (CSMA/CA) to reserve the channel for the entire duration of DATA transmission (collision avoidance scheme). In order to eliminate the collision among DATA packet, these both collision avoidance and contention resolution schemes was implemented in IEEE 802.11 DCF MAC protocol.

\subsubsection{Binary Exponential Back-off (BEB)}

According to IEEE 802.11 DCF MAC protocol, a node which has new packet ready to transmit will acquires the channel to be idle for Distributed Inter-Frame Space (DIFS) time interval then the node will proceed with transmission. Otherwise, if the busy channel is detected, the node refrain its transmission until the ongoing transmission completes and sense the channel again until it become idle for DIFS. Once the channel become idle for DIFS, instead immediately transmit the packet, it will initializes a back-off timer [5].

By using the back-off mechanism, the transmission will be deferred for randomly selected intervals to minimize the probability of collision with other waiting nodes. Thus, this mechanism is useful to avoid the channel capture when two or more nodes wait to access the channel at the same time. This is to avoid two or more nodes seize the channel immediately upon completion of ongoing communication on channel [6].

In the DCF protocol, each node chooses the backoff interval (BOinterval) uniformly between the ranges of $[0, \mathrm{CW}]$. The value of Contention Window $(\mathrm{CW})$ is based on number of retransmission happens due to failed transmission. In order to prevents the contention window from expand too large or reduce too small two boundaries known as $\mathrm{CWmin}$ and CWmax are introduced in IEEE 802.11 standard. The CW is dynamically controlled by the back-off algorithm, namely Binary Exponential Back-off (BEB) in the IEEE 802.11 DCF scheme [8].

According to BEB algorithm, for first attempt, when the packet is fresh, the value of $\mathrm{CW}$ set to be equal to minimum contention window (CWmin). Every time when the node experiences a packet collision (i.e. unsuccessful transmission), it will be increased exponentially according to $2^{\mathrm{m}} \mathrm{CWmin}$, until the value reaches maximum contention window (CWmax). After the successful transmission, IEEE 802.11 DCF requires the nodes reset their $\mathrm{CW}$ to minimum value so that the winning nodes can get a better chance to involve in subsequent packet transmission. When the channel is sensed idle, then the random back-off timer decrement accordingly. Otherwise, when the channel is sensed busy, then the random back-off timer will freeze. The frozen back-off timer will start decrement again after the channel become idle for longer than a DIFS period. The node will begin its transmission immediately upon the backoff timer expires [5].

Two techniques are employed in IEEE 802.11 DCF for packet transmission. The basic access mechanism is also known as Carrier Sense Multiple Access (CSMA) is the earliest and pioneer mechanisms used for packet transmission. It is default mechanism where it is employs two-way handshaking technique. As improvement to the basic access mechanisms, an optional four-way handshaking technique known as Carrier Sense Multiple Access with Collision Avoidance (CSMA/CA) has been standardized. This mechanism employs extra signaling packet such as Request-To-Send/Clear-To-Send (RTS/CTS). 


\subsubsection{Basic Access CSMA Mechanism}

According to basic access CSMA mechanism, the node which is intended to transmit a DATA packet will listen to the channel first. If the channel is sensed idle for DIFS intervals then the DATA packet transmission will commence. Otherwise, if the channel is sensed busy, then the node will defers the transmission and wait until the ongoing transmission on channel is completed. Upon the idle channel detection, instead immediately initiates the DATA transmission, the node will further defer its transmission for an additional DIFS time period following by back-off period as described in previous section.

All other nodes in the range of sender will overhear the DATA packet which is being transmitted. They will set their Network Allocation Vector (NAV) based on the duration field in the DATA packet. The duration field in the DATA packet is the sum of time is taken for the ACK packet transmission, DATA packet transmission and InterFrame Space (SIFS) intervals. The duration field is to provide information to all nodes in the network on how long the channel will be seized for the ongoing transmission. Based on this information, all nodes will refrain begin their transmission, thus the collisions can be reduced accordingly. Once the DATA packet successfully received from the source node, then the destination will waits for a SIFS interval and then an ACK packet will be transmitted back to the source node. This is to indicate that the DATA packet had successfully received. The ACK packet transmission is required in the communication to improve the reliability of the DATA packet that has been transmitted $[5,6]$.

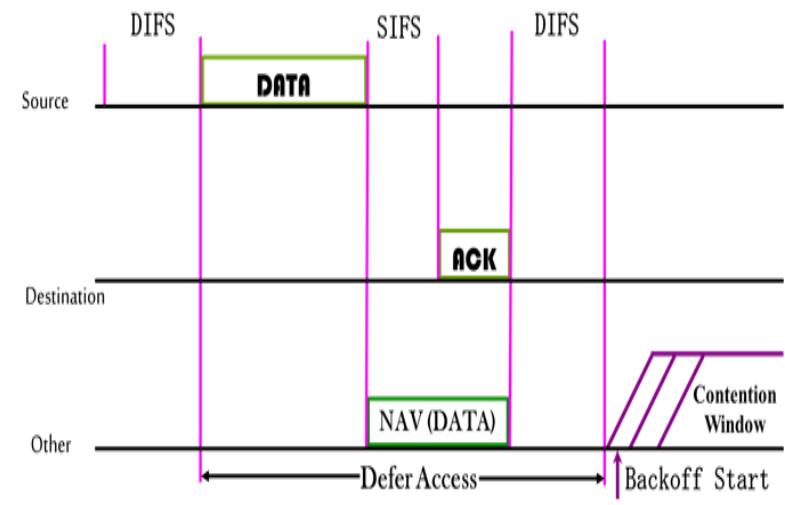

Fig 4: Basic Access Mechanism

Figure 4 illustrates channel negotiation method formed when implemented CSMA basic access mechanism. This mechanism is fully operating based physical carrier sensing method. This mechanism introduces well known problems which is called as hidden node $[9,10,11]$. When the nodes which are out of communication range between each other are communicating at the same time to the common receiver, this transmission will results in the collision. To solve this problem, an improvement to the basic access mechanism is proposed. The improved version is called as CSMA/CA four-way handshake access mechanism which is operates based on virtual carrier sensing.

\subsubsection{Carrier Sense Multiple Access with Collision}

\section{Avoidance (CSMA/CA) Mechanism}

CSMA/CA is the contention based MAC protocol and it is widely implemented protocol in wireless networks. The CSMA/CA is also defined in the IEEE 802.11 standard [1]. This protocol is an enhanced version of FAMA which was proposed to sense the carrier (CS) prior initiates the transmission. A sender node which is implemented with this protocol will sense the channel to check the transmission activities on channel. The node will proceeds with its transmission if the channel is sensed idle for a particular amount of time so called Distributed Inter-Frame Space (DIFS). Otherwise, if the channel is sensed busy, then it will defer its transmission until the channel is free for the transmission [15].

Upon idle channel detected, instead transmit the packet immediately, the sender node will choose back-off interval randomly as explained in previous section. If the channel is idle, then the back-off interval will start decrement otherwise if the channel is busy, then the back-of interval will freeze. According to back-off procedure, the frozen back-off interval will start decrement again after the channel is sensed idle for longer than DIFS. The sender node transmits the packet immediately when its back-off interval expires or reaches zero [16].

The hidden node problem is solved by CSMA/CA protocol by using RTS and CTS control packets. After the proper back-off and physical carrier sensing process, instead transmitting the DATA packet immediately, the sender node will reserve the channel by sending out RTS control packet to the destination node. After successfully received the RTS control packet, the destination node will remain silent for a SIFS interval, then responds with CTS control packet to the sender node. After successfully received the CTS control packet, the sender node will remain silent for the time interval corresponding to a SIFS and then it will begin the DATA packet transmission. All neighboring nodes which overhearing RTS/CTS control packets will defer themselves from sending out any packet until the predicted transmission period which as indicated in duration field of control packets is completed. This is called as Virtual Carrier Sensing method or most of time it is referred as Network Allocation Vector (NAV). This mechanism will be explained later in next section. Figure 5 illustrated the four- way handshake access method $[5,6,17]$. 



Fig 5: IEEE 802.11 DCF Four way handshake access method

Once the DATA packet successfully received, then the destination node will remain silent for the time interval of SIFS and then initiates an acknowledgment (ACK) control packet. The ACK control packet is used to improve the reliability of DATA packet reception. The priority for the nodes sending an ACK packet is higher than the nodes who want to initiates a new packet transmission because the duration of SIFS is shorter than DIFS. If the ACK is not received by source node (i.e. ACK_timeout), then the DATA packet is assumed lost and a retransmission according to BEB algorithm will be scheduled.

With the use of RTS-CTS control packets, the collisions caused by the hidden nodes can be eliminated. However, this method requires additional bandwidth which is utilized to transmit RTS and CTS control packets. The throughput achieved by CSMA/CA access method much better compare to basic access mechanism even though it introduced the additional overhead caused by RTS/CTS exchange. This is due to its effectiveness in minimizing the collision which is caused by hidden nodes $[10,11,18]$. In the presence of hidden nodes, the use of RTS/CTS can prevent the collision thus improve the performance of the network.

\subsubsection{Virtual Carrier Sensing Mechanism}

The virtual carrier sensing mechanism is used in CSMA/CA protocol to schedule larger DATA packets in a contentionfree manner. This mechanism also prevents the "hidden node" problem by distributing the RTS and CTS control packet to the nodes in the vicinity of both source and destination. This is possible with the help of Network Allocation Vector (NAV). All neighboring nodes which are overhear the RTS or the CTS control packets will update their NAV based on the Duration/ID field of the corresponding RTS and CTS control packet. This duration is to indicate for how long the node should defer its transmissions on the channel. The duration which is set in the RTS control packet is equal to the time is taken for transmit the CTS packet, DATA packet, ACK packet and plus with three SIFS intervals. The duration which is set in the CTS control packet is equal to the time is taken for transmit the DATA packet, ACK packet and plus with two SIFS intervals. Thus, virtual carrier sensing is used to silence the neighbors of active transmitter and receiver nodes for particular amount of time until the ongoing transmission completes. For the example, as shown in Figure 6, the neighbors of the source and destination are silence by RTS and CTS respectively.

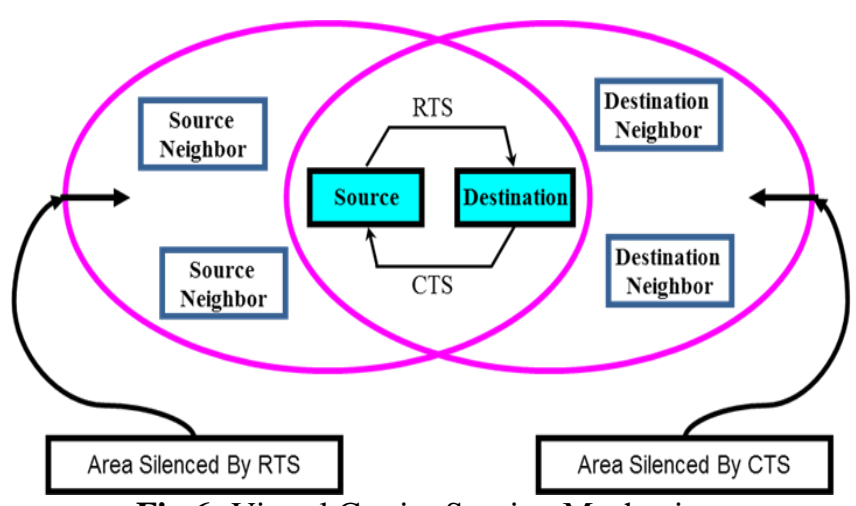

Fig 6: Virtual Carrier Sensing Mechanism

The use of RTS/CTS control packets mechanism prevent the larger DATA packet from involve in collision. For example, if two or more nodes initiate the packet transmission at the same time, then it will lead to the collision. However, if both transmitting nodes use the RTS/CTS mechanism before the DATA packet transmission could commence, thus the collision only will be occurred among RTS control packets. Therefore, the DATA-DATA collisions can be prevented through the usage of RTS/CTS mechanism in DCF CSMA/CA protocol. The collision among the RTS and CTS control packets are acceptable in the network because their sizes are too small. Thus the bandwidth loss due to this collision is small compare to DATA-DATA collision. This approach improves the system performance by reducing the bandwidth loss $[5,18]$.

\section{CONCLUSION}

In wireless network, Medium Access Control (MAC) protocol plays an important role to regulate an access to all nodes. This paper presents an overview and a survey of the research work conducted on MAC layer that specifically focused on contention and collisions issues in wireless network. In this article we have discussed the characteristics and operating principle of different MAC protocols and explained how the proposed MAC protocols solve contention and collision issues. Even though several MAC protocols have been proposed to date, but preventing the collision completely are still not fully satisfied yet. Therefore, still a lot of work still need to be done in working out an efficient MAC protocol that could solve on contention and collision and issues completely.

\section{ACKNOWLEDGEMENT}

The authors would like to take this opportunity to thanks those who are contributes directly or indirectly in completion of this article and also for their constructive comments. In addition, the authors also would like to express our gratitude to Universiti Teknikal Malaysia Melaka (UTeM) for the support and encouragement. 


\section{REFERENCES}

[1] IEEE, Institute of Electrical and Electronics Engineering "IEEE Standard for information Technology - Telecommunications and Information Exchange between Systems - Specific Requirements" - Part 5: Token Ring Access Method and Physical Layer Specifications. IEEE, New York, 1998.

[2] Fullmer, C.L. \& Garcia-Luna-Aceves, J.J. Floor Acquisition Multiple Access (FAMA) for packet radio networks. In Conference on Application, Technologies, Architectures and Protocols for Computer Communication (SIGCOMM), pp. 262273. 1995.

[3] Bharghavan,V., Demers, A., Shenker,S. \& Zhang, L. MACAW:A Media Access Protocol for Wireless LANs. Proc.ACM SIGCOMM'94, pp.212-225. 1994.

[4] Karn, P. MACA- A New Channel Access Method for Packet Radio.ARRL/CRRL Amateur Radio 9th Computer Networking Conference,pp.134-140. 1990.

[5] Rahim, N. Javaid, M. Aslam, Z. Rahman, U. Qasim, and Z. A. Khan, "A Comprehensive Survey of MAC Protocols for Wireless Body Area Networks," in Proceedings of the International Workshop on BioSensing, Processing, Application and Networking, Victoria, Canada, 2012.

[6] De Domenico, E. Strinati, and M.-G. Di Benedetto, "A Survey on MAC Strategies for Cognitive Radio Networks," IEEE Communications Surveys and Tutorials, vol. 14, no. 1, pp. 21-44, 2012.

[7] N. Faruk, A. Ali and M. Gumel, "Interference Mitigation MAC Protocol for Cognitive Radio Networks," Wireless Engineering and Technology, vol. 3, no. 2, pp. 63-71, 2012.

[8] J. Marinho and E. Monteiro, "Cognitive radio: survey on communication protocols, spectrum decision issues, and future research directions," Wireless Networks, vol. 18, no. 2, pp. 147-164, 2012.

[9] F. V. Gallego, J. Alonso-Zarate, C. V. Verikoukis, and L. Alonso, "A survey on prototyping platforms for the development and experimental evaluation of medium access control protocols," IEEE Wireless Communications, vol. 19, no. 2, pp. 74-81, 2012.

[10] A. Tsertou and D.I. Laurenson, "Revisiting the Hidden Terminal Problem in a CSMA/CA Wireless Network," IEEE Transactions on Mobile Computing, vol. 7(7), pp. 817-831, October 2008.

[11] Alam, M.D.; Hussain, M.A.; Kwak, K.S. Neighbor Initiated Approach for Avoiding Deaf and Hidden Node Problems in Directional MAC Protocol for AdHoc Network. Wirel. Netw. 2013, 19, 933-943.

[12] Tinnirello, G. Bianchi, P. Gallo, D. Garlisi, F. Giuliano, and F. Gringoli, "Wireless MAC processors: Programming MAC protocols on commodity Hardware," in Proceedings of the IEEE Conference on Computer Communications, Orlando, Florida, USA, 2012.

[13] J. C. O’Sullivan, P. di Francesco, U. K. Anyanwu, L. A. SaSilva, and A. B. MacKenzie, "Multi-hop MAC Implementations for Affordable SDR Hardware," in Proceedings of the IEEE Symposium on New
Frontiers in Dynamic Spectrum Access Newtorks, Aachen, Germany, 2011.

[14] J. Ansari, X. Zhang, S. Gaikwad, and P. Mah“ onen, "Exploring MAC Parallelization on Software Defined Radio Platforms," in Proceedings of the IEEE International Conference on Sensing, Communication, and Networking, New Orleans, USA, 2013.

[15] Vigneswara Rao, Gannapathy., Suaidi, Mohamad Kadim., Johal, Muhammad Syahrir Bin., Chuan, Lim Kim., Ramli, Nordin., Mohamad, Hafizal. "A Smooth Forwarding Operation in Wireless Mesh Network," in IEEE 10th Malaysia International Conference on Communications (MICC), pp. 83-87. 2011.

[16] Vigneswara Rao Gannapathy, Tuani Ibrahim, Ahamed Fayeez, Zahriladha Zakaria, Abdul Rani Othman, Nur Qalbi Jalaudin, "A review on various types of Software Defined Radios (SDRs) in radio communication", IJRET: International Journal of Research in Engineering and Technology eISSN: 2319-1163, pISSN: 2321-7308, Volume: 03 Issue: 12, Dec-2014

[17] Vigneswara Rao Gannapathy, Ahamed Fayeez Bin Tuani Ibrahim, Zahriladha Bin Zakaria, Abdul Rani Bin Othman, Mohamad Kadim Bin Suaidi, "Alleviate Exposed Node Issues In Wireless Mesh Network (WMN) Using A Novel Approach Of Concurrent Medium Access Control (C-MAC) Protocol”, IJRET: International Journal of Research in Engineering and Technology eISSN: 2319-1163, pISSN: 2321-7308, Volume: 03 Issue: 11, Dec-2014

[18] Vigneswara Rao Gannapathy, Ahamed Fayeez Bin Tuani Ibrahim, Zahriladha Bin Zakaria, Abdul Rani Bin Othman, Anas Abdul Latiff, "An Enhancement Of RTS/CTS Control Handshake In CSMA/CA Based MAC Protocol For An Efficient Packet Delivery Over Multi-hop Wireless Mesh Network (WMN)", IJRET: International Journal of Research in Engineering and Technology eISSN: 2319-1163, pISSN: 2321-7308, Volume: 02 Issue: 10, Dec-2013.

[19] A.T.I Fayeez, V.R Gannapathy, S. S. S Ranjit, S.K. Subramaniam, Ida S.Md Isa, "Throughput Analysis Of Energy Aware Routing Protocol For Real-Time Load Distribution In Wireless Sensor Network (WSN)", IJRET: International Journal of Research in Engineering and Technology eISSN: 2319-1163, pISSN: 2321-7308, Volume: 02 Issue: 11, Nov-2013.

[20] Abdulaziz M. Ghaleb, David Chieng, Alvin Ting, Seh Chun Ng, Ayad Abdulkafi, Kim-Chuan Lim, HengSiong Lim, "Preservation of QoS Across Hybris LTE-WLAN Routers", IET International Conference on Information and Communications Technologies (IETICT), Beijing, April 2013.

[21] Abdulaziz M. Ghaleb, David Chieng, Alvin Ting, Kae Hsiang Kwong, Kim-Chuan Lim, Heng-Siong Lim, "Throughput Analysis of IEEE 802.11n Using OPNET", IET International Conference on Wireless Communication and Application (ICWCA 2012), ISBN: 978-1-84919-550-8, October 2012. 


\section{BIOGRAPHIES}

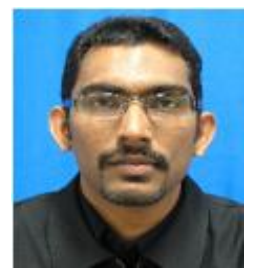

Vigneswara Rao Gannapathy currently serving as a lecturer in Universiti Teknikal Malaysia Melaka (UTeM) and he actively involves in research activities which is related to Electronics and Wireless Communication. His research interest and direction has focused on $5 \mathrm{G}$ Wireless Communication Systems, IoT and Big Data that emerged as a key technology for next - generation Wireless Communication.

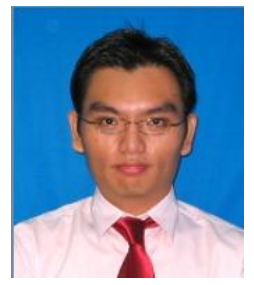

Lim received his B.Eng (2003), MSc. (2005), and PhD. (2009) degrees from the Faculty of Arts, Computing, Engineering and Sciences, Sheffield Hallam University., UK. From 2005 he was a research assistant in the Mobile Machines and Vision Laboratory (MMVL) at Sheffield Hallam University. He is currently a senior lecturer at the FKEKK, UTeM. Malaysia

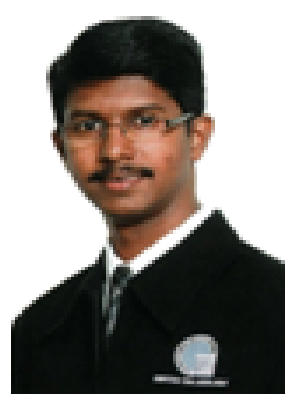

Siva Kumar Subramaniam graduated with B.Eng (Hons) and M.Sc in Electronics Engineering from Universiti Teknikal Malaysia, Melaka in 2006 and 2009, respectively.His research interest is in wireless sensor network for long range data transmission using IEEE 802.11 and IEEE 802.15.4 standards.

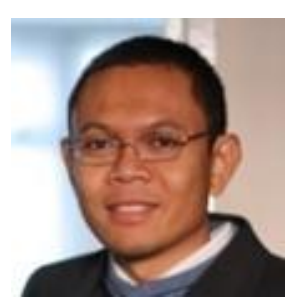

Dr. Zahriladha Zakaria, PhD, MIEEE, BEM, Grad IEM is currently working as a lecturer at the Faculty of Electronic and Computer Engineering, University Teknikal Malaysia Melaka. (UTeM). His research interests include a variety of microwave device development such as planar and nonplanar microwave filters, amplifiers and antennas. 